Journal of Social Sciences 6 (4): 515-522, 2010

ISSN 1549-3652

(C) 2010 Science Publications

\title{
Many Sources, One Theme: Analysis of Cyberbullying Prevention and Intervention Websites
}

\author{
Rebecca Ahlfors \\ Massachusetts School of Professional Psychology, \\ Mental Health Clinician at LUK Crisis Center, Inc., United States of America
}

\begin{abstract}
Problem statement: Cyberbullying represents the expansion of traditional bullying into the electronic realm. As the problem of cyberbullying typically occurs via the Internet, many cyberbullying prevention and intervention resources have been made available online. The purpose of this qualitative study was to determine general characteristics of cyberbullying prevention and intervention websites. This study was conducted as a preliminary survey of seventeen independent cyberbullying prevention websites. Approach: Categories for analysis were developed following consultation with three psychologists, one of whom specializes in online psycho educational interventions. Each of the seventeen cyberbullying websites was then reviewed for the same variables of target audience, main topics, use of published research and methods for relaying information. Results: Fourteen of the websites are oriented toward informing parents and caregivers while seven address latency aged children, eight address children ages eleven and twelve and eleven address adolescents. Nine of the websites address educators and six provide information for law enforcement professionals. The main topics consist of cyberbullying prevention and Internet safety tips, availability of a commercial product, methods for managing cyberbullying, signs a child is experiencing cyberbullying, information regarding the motivations for cyberbullying and miscellaneous but related topics. Sixteen of the seventeen reviewed websites present information comparable to the information found in empirically validated studies and ten of the websites list references to the published literature on the site. Methods for relaying information include text only, interactive formats, videos, personal stories, message board/blogs and newsletters. Conclusion/Recommendations: Psychological research showed that victims of cyberbullying believe they should manage the problem on their own and rarely confide in their parents. The results of analysis of the target audiences suggested that there is a disconnection between Internet based cyberbullying interventions and the main target audiences. To address this problem, future research should include evaluation of the accessibility and effectiveness of cyberbullying psycho educational websites designed for youth. Other areas to be researched include the potential negative consequences of online cyberbullying interventions and the need for Internet based cyberbullying prevention websites designed for cyberbullies. A definite area in need of research is on the efficacy of Internet based cyberbullying psycho educational sites in decreasing incidences of cyberbullying.
\end{abstract}

Key words: Cyberbullying, intervention websites, children and adolescents, electronic bullying

\section{INTRODUCTION}

Technology is rapidly developing. Countless pieces of information are added to the Internet each day. Advances in technology are often marketed toward older children and adolescents. These have provided new venues for positive peer connections, through both locally-based and World Wide social networking forums. Youth are turning to the Internet for expanded social connections. One unfortunate development from the development of communications technology is the emergence of cyberbullying, also referred to as
"Electronic Bullying". Both can be defined as "An aggressive, intentional act carried out by a group or individual using electronic forms of contact, repeatedly and over time against a victim who cannot easily defend him or herself" (Smith et al., 2008).

Cyberbullying occurs through the use of "real time" methods such as Instant Messaging (IM), chatroom communications, cellular telephone tools such as text messaging ("texting") and forwarding electronic images. It can occur in delayed messaging such as electronic mail (email) and website postings. 
Cyberbullying assaults can occur at any time and in any place that the target has access to electronic communication. Messages or images sent electronically can reach multiple people concurrently, increasing the number of witnesses to the degradation of the target. Perceived anonymity of the cyberbully adds fear of the unknown to the experience of the target (Kowalski and Limber, 2007).

A "cyber bully" is a person who uses electronic means to cause emotional harm to another person. The perpetrator demonstrates a pattern of behavior of repeatedly engaging in online acts to humiliate another; either the same target or targets each time, or against random, unknown targets.

Gender does not appear to impact the frequency of engagement in cyberbullying behaviors ( $\mathrm{Li}, 2006)$. Cyber bullies are predominantly Caucasian and are frequently the same students who bully peers in person (Hinduja and Patchin, 2008; Li, 2006; Luan et al., 2008). According to Hinduja and Patchin (2008), offline bullies are "five times" as likely to bully others online. While offline bullies are more likely to engage in cyberbullying, victim reports indicated that cyber bullies are known bullies from school only $50 \%$ of the time, with strangers and siblings making up the other cyberbullying groups (Kowalski and Limber, 2007).

Technology provides a suitable medium for aggressive behaviors in that online communications often appear to be anonymous and therefore untraceable. The belief that one cannot be identified with online behavior leads to increased disinhibition of behavior (Mason, 2008). The cyber bully may engage in bullying behaviors online that they would never engage in when in person.

The targets or victims of online bullying are also referred to as "cyber targets" or "cyber victims". Males and females experience cyberbullying at an even rate (Slonje and Smith, 2008; Smith et al., 2008), with name-calling and insults being the most frequent form of electronic abuse experienced by the victims (Juvonen and Gross, 2008). Ongoing cyberbullying assaults have negative consequences for the victims. Half of the youth who were targeted online by the same person who bullied them in school reported feeling emotionally distraught by the online teasing (Ybarra et al., 2007). Youth who experience cyberbullying are more likely to carry weapons to school, to skip school or be suspended from school and to use alcohol or other substances (Ybarra et al., 2007).

Certain risk factors increase the likelihood that a student will experience cyberbullying. About $88 \%$ of cybervictims used the computer at least one time a week $(\mathrm{Li}, 2007)$. The more time a student spends on line, the more likely he or she is to experience cyberbullying (Hinduja and Patchin, 2008; Juvonen and Gross, 2008). The likelihood of experiencing cyberbullying also increases with the use of Instant Messaging (IM) and webcams (Juvonen and Gross, 2008).

As with other forms of abuse, the victims of cyberbullying often attempt to avoid disclosure of the electronic abuses they are experiencing. The reluctance of victims to report experiencing cyberbullying appears to be a cross cultural phenomenon. A study of Swedish students who had experienced cyberbullying found that $50 \%$ of students did not report the cyberbullying and only $8.9 \%$ reported the abuse to their parents (Slonje and Smith, 2008). Similar results have been found in a study of cyberbullying victims in the United States, with approximately $50 \%$ of victims not telling anyone and $15.5 \%$ disclosing to their parents (Smith et al., 2008). Many students reported feeling a need to manage or "deal" with cyberbullying individually (Juvonen and Gross, 2008). Aside from the feelings of shame that often accompany being the victim of bullying, many children and adolescents fear that if they report the cyberbullying to their parents, they will be restricted in their use of electronics (Juvonen and Gross, 2008).

Despite their attempts to intercede, parent interventions are often insufficient due to youth's reluctance to disclose experiencing electronic bullying to their parents (Ybarra et al., 2007).

Since youth often fail to confide in parents regarding cyberbullying, the school systems are asked to intervene by developing clear and consistent guidelines regarding the use of electronic communication (Mason, 2008). While consistent guidelines may be effective in decreasing cyberbullying incidents during school hours, youth are even less likely to disclose cyberbullying abuse to teachers and school personnel than they are to disclose to their parents (Slonje and Smith, 2008; Smith et al., 2008). While the problem of cyberbullying is addressed by the school systems, individual cyberbullying victims may remain uncertain on how best to manage cyberbullying when it occurs.

Given that youth tend to keep cyberbullying incidents to themselves, combined with the amount of time today's youth spend using electronic tools, the Internet becomes the next logical method for reaching children and adolescents around the issues related to cyberbullying. Many students spend significant amounts of time online and may need less encouragement to use computer-based intervention programs. Additionally, a cyber-victim who may be 
unwilling to identify solutions to the cyberbullying through communication with an adult may feel more comfortable researching solutions on the Internet.

There are a number of cyberbullying prevention and intervention websites that have been developed and are currently available on the World Wide Web. While specific cyberbullying prevention programs, including online programs, have been field tested already, analysis of the general information and themes available through online cyberbullying prevention and intervention websites has not yet been conducted. This study uses qualitative analysis of seventeen cyberbullying prevention and intervention websites in order to show what information about cyberbullying is currently available online as well as to identify those areas that are not being addressed as readily.

Many websites vary in quality and accuracy of the information being presented. It is important to identify and understand the various states of available websites that address cyberbullying. As part of the analysis of themes in these seventeen websites, analysis of the information contained in each website was conducted in comparison to the information provided by the professional literature. Each website was also reviewed for evidence of use of the professional literature with regard to cyberbullying.

\section{MATERIALS AND METHODS}

Research design: In this qualitative analysis, forty cyberbullying websites were selected by searching for terms related to cyberbullying over an Internet search engine. The forty websites were divided into two categories; web pages that were embedded within larger informational websites and those websites dedicated strictly to cyberbullying and not included as part of general informational websites. In order to establish a clear baseline for comparison, the seventeen websites dedicated only to cyberbullying prevention and intervention were selected for further analysis.

The categories to be analyzed were developed through conversations with three psychologists, one of whom specializes in psycho educational website design. The analysis of the websites was conducted by the author. Even though objective criteria were used for analyzing the information in each category, some degree of subjective judgment was required in evaluating some data. It is important to note that this study is of qualitative design and useful for developing areas of future research rather than gathering statistically significant data. The criteria used in the assessment of the seventeen independent cyberbullying websites included:
- Target audiences

- Main topics addressed

- Methods for relaying information

- Inclusion of Reference to Published Research

\section{Procedures:}

Data collection: The websites reviewed were chosen through the use of Internet searches for the terms "cyberbullying", "cyber-bullying", "cyberbullying", "cyber victim", "cyberbullying prevention", "cyberbullying info" and "online harassment". Wellknown search engines were utilized. Since the websites reviewed are in the public domain, it was acceptable to use the information without permission.

The first category analyzed was Target Audience of the websites. The target populations of each website were determined by observing the listings of specific groups of people of the website. The different groups of people were often indicated by separate icons or "buttons" that would redirect the website user to information designed specifically for individuals of that category. The target audiences for the seventeen websites consisted of latency age children (ages 6-10), "tweens" (ages 11-12), Adolescents (ages 13-18), Parents/Caregivers, Educators and Law Enforcement professionals.

Main topics were defined by headings and links within the websites and are discussed in the results.

The methods for relaying information are divided into the categories of Text only, Interactive media programs (quizzes, games, songs, still cartoons), Videos including quotes from individuals, advertisement endorsements, skits, BLOG or message boards, Newsletters and Human Interest stories.

A website was considered to provide Reference to professional literature if the website contained a specific list of professionally reviewed references. The websites that did not provide a separate references list were analyzed for content based on a previously conducted review of the professional literature available concerning cyberbullying.

\section{RESULTS}

Data analysis: Seventeen independent websites dedicated to the topics of cyberbullying education, prevention and intervention were reviewed. These websites include:

- www.cyberbullying.org

- www.stopcyberbullying.org

- www.wiredsafety.org/index.html

- www.cyberbullying.us/ 
- www.cyberbully.org

- www.slais.ubc.ca.COURSES/libr500/04-05-

- www.cyberbullinginfo

- $\quad$ www.netsmartz.org/index.aspx

- www.isafe.org

- www.cyberbullyhelp.com

- www.cyberbullyalert.com

- www.cyberbully411.org

- www.cyberbullyingprevention.com

- www.wiredkids.org/

- http://bullyonline.org/

- www.online-bully.com/

- http://beenbulliedonline.com/

Target audience: The information in the reviewed cyberbullying websites most often addresses parents or caregivers, with fourteen of the websites including information for parents. Eleven websites address adolescents (ages 13-18). Nine of the websites provide information for Educators. Eight of the cyberbullying websites target "Tweens" (ages 11-12). Seven sites are designed for younger children (ages 6-10). Six of the websites have information for Law Enforcement professionals.

\section{Website topics:}

Prevention/safety tips category: Sixteen of the seventeen websites included a definition of cyberbullying that corresponds with definitions already established in the literature by cyberbullying experts. Seven of the websites incorporated either specific cyberbullying prevention tips and/or general Internet safety tips. A few examples of these safety tips include protecting passwords, keeping personal information off of the Internet and methods for reporting CBL (www.stopcyberbullying.org; www.cyberbully.org; www.slais.ubc.ca.COURSES/libr500/04-05-

st2/www/D_Jackson/index.htm;

www.cyberbullying.info; www.netsmartz.org; www.isafe.org; www.wiredkids.org).

Outreach/lesson plans category: Six of the websites included ideas for increasing outreach to others through peer interaction programs or through specific educational lesson plans. The peer interactions are designed primarily for adolescents whereas the lesson plans are designed for Educators (www.stopcyberbullying.org; www.wiredsafety.org; www.slais.ubc.ca.COURSES/libr500/04-05-

st2/www/D_Jackson/index.htm; www.isafe.org; www.cyberbullyhelp.com;

www.cyberbullyingprevention.com).
Commercial category: There were six websites designed around a commercial product. The products being sold included books on cyberbullying, educational cyberbullying prevention programs and computer monitoring software (www.cyberbully.org; www.isafe.org; www.cyberbullyalert.com;

www.cyberbullyingprevention.com; www.onlinebully.com).

Intervention/response category: Five of the websites provided specific directions on ways to manage cyberbullying. The information was provided for victims, witnesses, parents and/or educators (www.cyberbulling.org; www.wiredsafety.org; www.cyberbullying.info; www.cyberbully411.org; www.online-bully.com).

Forms/technology: The specific forms of cyberbullying and the technology used for cyberbullying acts are addressed in five of the websites. Some forms cyberbullying can take include insults, threats and social exclusion. Cyberbullying can occur through social networking pages, Instant Messaging (IM's), email and over cellular telephones (www.cyberbullying.org; www.stopcyberbullying.org; www.cyberbullying.info;

www.cyberbullyingprevention.org; www.onlinebully.org).

Symptoms of cyberbullying: Four of the websites inform adults of signs that their child may be experiencing cyberbullying. Symptoms listed include behavioral withdrawal, hiding computer based communications, appearing upset following receipt of technological communication, depression, somatic complaints and decline in academic achievement (www.cyberbullying.org; www.stopcyberbullying.org; www.cyberbullying.us; http://bullyonline.org).

Why cyberbully: Three websites addressed theories regarding the motivations of youth for engaging in cyberbullying. Within these websites, the motivations of a cyberbully were listed as for "laughs", to gain power and to reduce anxiety, frustration and anger (www.stopcyberbullying.org; www.cyberbullying.us; www.cyberbully411.org).

Miscellaneous: This category was included to cover information related to cyberbullying, including cyberstalking, Internet predators, a cyber-tip line, Internet Safety Tips training for seniors, alternative activities to technology and the signs and symptoms of 
depression (www.wiredsafety.org; www.netsmartz.org; www.isafe.org; www.cyberbullyhelp.com; www.cyberbully411.org; $\quad$ www.wiredkids.org; http://beenbulliedonline.com).

References to published research: Ten websites identified specific scholarly research that had been used in their development. The research used in these websites is acknowledged either at the bottom of one of the main pages or as a separate page within the website. Sources for the research included psychological research on prevention and intervention of cyberbullying, educational journals, legal sources, government endorsed research and the media (www.cyberbullying.org; www.stopcyberbullying.org; www.wiredsafety.org; www.cyberbullying.us; www.cyberbully.org; www.slais.ubc.ca,COURSES/libr500/04-05st2/www/D_Jackson/index.htm;

www.cyberbullying.info; www.cyberbullyhelp.com; www.cyberbully411.org;

www.cyberbullyingprevention.com).

There were three websites designed by the same individual, a lawyer who specializes in privacy and cybercrime law. Two of these websites did not indicate research on the WebPages (www.netsmartz.org; www.wiredkids.org), while the third website did acknowledge an extensive resource list (www.wiredsafety.org). Given that the same individual designed and supervises the moderation of all three websites, a resource list was provided for one of the websites and the content of all three websites is similar, an additional listing of references on all three websites was likely considered unnecessary. While not all of the websites cited research specifically, sixteen of the seventeen websites provided information that is consistent with the information given in the professional literature.

One website presented primarily as a "blog" and did not cite references of published research. (http://beenbulliedonline.com).

Information delivery methods: The Written Text category was developed for those websites that did not utilize any other information delivery methods except for written text. Just two of the websites used written text only as an information delivery method (www.cyberbullyhelp.com; www.wiredkids.org).

The Message board/BLOG category was developed to indicate whether a cyberbullying website also included an online message board or blog discussion section. It is important to note that the presence of a BLOG typically indicates a need for a database to which information can be sent regarding cyberbullying incidences. Eleven of the websites had a messageboard or BLOG (www.cyberbullying.org; www.wiredsafety.org; $\quad$ www.cyberbullying.us; www.cyberbully.org; www.isafe.org; www.netsmartz.org; www.cyberbully411.org; www.cyberbullyingprevention.com; http://bullyonline.org; http://beenbulliedonline.com).

The Human Interest/Stories category was developed to for websites that include personal narratives of individuals involved with cyberbullying. These stories often consisted of several sentence quotes up to several paragraph stories and were most frequently told from the perspective of victims, witnesses and parents. There were very few quotes from cyberbullies. Some of the quotes were used to promote or endorse the sale of cyberbullying prevention and intervention products. Eight of the websites use Human Interest stories (www.cyberbullying.org; www.cyberbullying.us; www.netsmartz.org; Http://bullyonline.org; Http://beenbulliedonline.com).

The Interactive category covers any websites that require the user to engage in an activity beyond reading simple text. Interactive engagement with the website includes quizzes, online games, songs and comic strips as information delivery methods. There were six websites that use Interactive information delivery (www.stopcyberbullying.org; www.wiredsafety,org; www.cyberbullying.us;

www.slais.ubc.ca.COURSES/libr500/04-05-

wt2?www/D_Jackson/index.htm;

www.cyberbullying.info; www.netsmartz.org).

Five of the websites were listed in the Video category. These websites use videos that include skits, endorsements for products or psycho educational tools and the experiences of people involved with cyberbullying www.cyberbullying.info; (www.cyberbullying.us; www.netsmartz.org; www.isafe.org; www.online-bully.com). One website has developed a program of Internet safety education based around videos for purchase (www.isafe.org).

The Newsletter category was developed to indicate online newsletter availability. Only two of the websites had an online newsletter (www.cyberbullying.us; www.cyberbullyingprevention.com).

\section{DISCUSSION}

Of the cyberbullying websites reviewed, the most frequently addressed population is Parents/Caregivers. 
However, according to the research on cyberbullying interventions, parents are often not aware of their children's and adolescents' cyberbullying experiences, as children do not confide in their parents about experiencing cyberbullying assaults. According to these results, the populations most in need of Internet based cyberbullying interventions are not the most frequently addressed. Whereas it is useful to educate parents regarding cyberbullying, it is apparent that the websites need to increase the focus on children and adolescents.

Adolescents are the second most frequently addressed groups of the cyberbullying prevention websites. It is uncertain whether or not these websites are increasing awareness for adolescents around cyberbullying and further research needs to be conducted.

The websites that promote commercial products are designed for parents. It is undeniable that capitalism plays a role in sustaining the Internet. The information about the problem of cyberbullying is being disseminated in part by the developers of the cyberbullying prevention products. This has the potential to increase the awareness of cyberbullying in the adult population.

Of the websites reviewed, only one had a main focus as a "blog" (message board). While some websites used a messageboard in part of the design, the main content of this website consisted of communications on the "blog". This site contained links to information content, but the information provided was minimal, consisting of only two to three sentences. The target population of this website was not divided by age or profession and was instead directed towards people who have experienced bullying. The website invites people to describe their cyberbullying experiences in a public forum. Other users of the messageboard respond to what is written. In a format such as this, the potential for further abuse is highly possible, with damage being done to participants before the moderator (s) are able to remove cyberbullying content. Additionally, since the responders are not necessarily professionals in managing bullying and cyberbullying, a messageboard user could receive ill advised suggestions. A website where people share experiences may be helpful to decrease the isolation targets can feel, however it is recommended that individuals who wish to share their experiences with bullying online use professionally designed and/or moderated websites.

The Information Delivery Method category was developed in anticipation of areas for consideration for future research. Of the reviewed websites, all include written text, but only two consisted of only written text.
Although not empirically addressed in this study, one hypothesis is that text only websites would be disinteresting to children, many adolescents and some adults. The websites that employ interactivity, videos and personal stories have the potential to attract and hold the attention of users for longer time periods. The longer an individual remains on a website, the longer that person will be exposed to the information presented. Interactive methods for information delivery will sometimes provide information in a less direct manner for the sake of developing an entertaining activity. While interactive engagement may hold an individual's attention to the subject matter, the meaning and learning associated with the activity may be less effective.

The results of this analysis do not provide information on the effectiveness of one information delivery method over another. Should Internet based cyberbullying interventions continue to be utilized, future research should address the efficacy of Interactive methods, videos and personal stories for increasing learning of cyberbullying prevention information, as well as website user preference for the different methods of information delivery.

Many of the self report comments provided on these websites were from parents, adolescents and children, involved with cyberbullying as targets, witnesses, or cyberbullies. Less frequently, quotations included promotion of cyberbullying prevention and intervention products or programs.

While many organizations and websites use online newsletters, two of the seventeen cyberbullying websites reviewed promoted signing up for a newsletter. One hypothesis for this result is that the maintenance of an online newsletter entails additional research and resources and those online newsletters are not a cost effective method for delivering information. However, online newsletters that are delivered to individuals' email boxes on a consistent basis may serve as a reminder to Internet users of the issue of cyberbullying.

Due to the limited time and resources available to the researcher, some important categories may have been excluded from the analysis of the websites. Future research could identify other beneficial categories for cyberbullying website analysis.

Recommendations for future research: Many of the websites addressing cyberbullying prevention and intervention have been developed by professionals who use empirically validated information. These websites have been designed to provide accurate information in an accessible manner. Not all psychoeducational 
websites give evidence of the use of professional literature in their design and it is possible that some websites that appear to present researched information are not developed from scientifically validated studies. Websites should provide a clear and easily accessible list of concrete references, so that users will be more able to identify those websites that contain valid information.

Cyberbullying prevention websites often include information on Internet safety tips. It is conceivable that an individual engaged in cyberbullying could potentially use this information to become more effective in avoiding detection when cyberbullying others. Reporting about the forms cyberbullying can take could also give cyberbully new ideas for their abuse of others. Awareness of the negative consequences cyberbullying has on victims could serve to reinforce cyberbullying behavior. As the Internet becomes more of a resource for cyberbullying prevention, it will be important to investigate the negative potential impact of these websites.

Some of the websites reviewed provided guidance for those who witness cyberbullying or who become cyberbullies in reaction to the cyberbullying of others Children and adolescents who engage in unprovoked cyberbullying were not listed as a separate target audiences.

Out of the seventeen websites that were reviewed, none were specifically designed for individuals who engage in cyberbullying behaviors. This indicates a significant lack of Internet based interventions for youth engaging in cyberbullying. A cyberbullying website specifically designed for cyberbullies needs to be developed. It is possible that youth who cyberbully are not aware that their behaviors constitute bullying. These youth may not use such resources as they would not have the awareness that their online behavior is problematic. Additionally, cyberbullies may not be motivated to change their behaviors and may be less likely to access online resources as readily as cyberbullying victims would. It may be necessary to find alternative methods for engaging these populations. Should a website specifically addressing cyberbullying children and adolescents be developed, it is recommended that the efficacy of this website be evaluated and adjustments made accordingly, over time.

A review of the cyberbullying literature indicates that most children and adolescents will not talk to their parents when they are being cyberbullied. In the analysis conducted here, the Parent category for target audience was the most frequently addressed with fourteen of the websites. However, educating the parents about cyberbullying will not automatically encourage children and adolescents to tell their parents of instances when they have been cyberbullied. It may be important for website designers to increase the focus on helping children and adolescents manage cyberbullying independently along with continuing to encourage cyberbullying victims to tell their parents about their cyberbullying experiences. It will be helpful to determine if websites that use interactive methods and videos are more able to gain and hold the attention of younger website users without sacrificing clarity or breadth of covered information. While children and adolescents turn more and more frequently to the Internet for the information they need, we need to provide those answers in a way that will teach children and adolescents that they are capable of stopping cyberbullying.

Not enough attention has been given to expanding knowledge about the effectiveness of Internet based cyberbullying prevention and intervention websites in general. It is especially difficult to assess the efficacy of these websites. A starting point would be to determine how many people are accessing the cyberbullying prevention websites, combined by soliciting opinion from users as to whether the information was of help or not.

\section{CONCLUSION}

Cyberbullying prevention and intervention websites have been developed to address the growing problem of cyberbullying. The purpose of this qualitative study was to analyze seventeen cyberbullying prevention websites to identify information currently available online regarding cyberbullying. The results indicate that although the victims of cyberbullying will typically not report the abuse to parents, more of the reviewed websites addressed parents and guardians than children, "tweens" and adolescents. The main topics addressed by these websites include safety and cyberbullying prevention, cyberbullying prevention products for purchase, cyberbullying interventions, signs a child is being cyberbullied, the motivations for cyberbullying and other topics related to cyberbullying. Methods for relaying information include written text only, interactive formats, videos, personal stories, message board/blogs and newsletters. Ten of the websites list references to published literature and sixteen of the websites provide accurate information.

As a result of this analysis, missing information was also identified. None of the seventeen reviewed websites are designed specifically for youth who actively engage in cyberbullying others. Additionally, it 
is unknown if cyberbullying prevention websites could have a possible detrimental impact by providing information to cyberbullies.

What is the efficacy of cyberbullying education, prevention and intervention websites in decreasing incidences of cyberbullying? What can be done to encourage children and adolescents to access the resources already available on the Internet with regard to cyberbullying prevention? These questions should be used to assist in guiding future research of Internet based cyberbullying interventions.

\section{ACKNOWLEDGMENT}

I wish to acknowledge Kenneth Hopkins, PsyD, Wynn Schwartz, PhD and Rory Stern, PsyD for their assistance in the conceptualization of this project. Ronald E. Cobb, PhD helped with data interpretation and served as the initial editor for this article. I also wish to thank Rita Zeffert, MEd and Adrian Zeffert for additional assistance. Lastly, I would like to thank Elizabeth Englander, $\mathrm{PhD}$ for inviting this submission.

\section{REFERENCES}

Hinduja, S. and J.W. Patchin, 2008. Cyberbullying: An exploratory analysis of factors related to offending and victimization. Deviant Behav., 29: 129-156. DOI: 10.1080/01639620701457816

Juvonen, J. and E.F. Gross, 2008. Extending the school grounds?--bullying experiences in cyberspace. J. Schools Health, 78: 496-505. PMID: 18786042
Kowalski, R.M. and S.P. Limber, 2007. Electronic bullying among middle school students. J. Adolesc. Health, $41: \quad 22-30 . \quad$ DOI: 10.1016/j.jadohealth.2007.08.017

Li, Q., 2006. Cyberbullying in schools a research of gender differences. School Psychol. Int., 27: 157-170. DOI: $10.1177 / 0143034306064547$

Li, Q., 2007. New bottle but old wine: A research of cyberbullying in schools. Comput. Hum. Behav., 23: 1777-1791. DOI: 10.1016/j.chb.2005.10.005

Luan, W.S., N. Siew F. and H. Atan, 2008. Gender Differences in the usage and attitudes toward the internet among student teachers in a public Malaysian University. Am. J. Applied Sci., 5: 689-697. DOI: $10.3844 / .2008 .689 .697$

Mason, K.L., 2008. Cyberbullying: A preliminary assessment for school personnel. Psychol. Schools, 45: 323-348. DOI: 10.1002/pits.20301

Slonje, R. and P.K. Smith, 2008. Cyberbullying: Another main type of bullying? Scand. J. Psychol., 49: 147-154. PMID: 18352984

Smith, P.K., J. Mahdavi, M. Carvalho, S. Fisher and S. Russell et al., 2008. Cyberbullying: Its nature and impact in secondary school pupils. J. Child Psychol. Psychiatry, 49: 376-385. PMID: 18363945

Ybarra, M.L., M. Diener-West and P.J. Leaf, 2007. Examining the overlap in Internet harassment and school bullying: Implication for school intervention. J. Adolesc. Health, 41: S42-S50. DOI: 10.1016/j.jadohealth.2007.09.004 